\title{
Simple Non-Aqueous Fabrication Route for Oleic Acid Capped Luminescent Cadmium Sulphide Quantum Dots at Relatively Low Temperature
}

\author{
Jayesh Patel $^{1,2}$, Frej Mighri $^{1,2 *}$, Abdellah Ajji ${ }^{1,3}$ \\ ${ }^{1}$ Center for Applied Research on Polymers and Composites, CREPEC; ${ }^{2}$ Chemical Engineering Department, Laval University, Quebec, \\ QC, G1K7P4 Canada; ${ }^{3}$ Chemical Engineering Department, École Polytechnique de Montréal, C.P.6079, Succ. Centre-Ville, Montreal, \\ QC, H3C 3A7 Canada. \\ E-mail: frej.mighri@gch.ulaval.ca
}

Received April 14 ${ }^{\text {th }}$ 2011; revised May 19 $9^{\text {th }}$ 2011; accepted May 30 ${ }^{\text {th }}, 2011$.

\begin{abstract}
In this communication, we report a simplistic non-aqueous fabrication route for oleic-acid capped luminescent cadmium sulphide quantum dots at low temperature. The resulting quantum dots, obtained without using any complicated reagents, are cubic in phase, monodispersed and have excellent optical properties. The simplicity of this fabrication route is also promising to develop other oleic acid-capped metal sulphide quantum dots at relatively low temperature.
\end{abstract}

Keywords: Semiconductor, CdS Quantum Dots, Quantum Confinement, XPS, Photoluminescence

\section{Introduction}

Metal chalcogenides has achieved significant attention in recent years and amongst them, nanoscale cadmium sulphide (CdS) has been considered as a very useful material for modern industrial applications [1,2]. CdS is one of the most important II-IV groups of semiconductors with a direct band gap of around $2.4 \mathrm{eV}$ at room temperature [3]. The size dependent properties of CdS Quantum dots (QDs) have been studied very frequently because of their potential application in photoelectric conversion devices [4], light-emitting diodes [5], nonlinear optics [6], and biological labeling [7]. Recently, CdS QDs are also considered as photocatalytic and nanoelectronic materials [2,8,9].

Morphology and size control of nanomaterials are key factors to improve their properties. Therefore, a variety of new fabrication routes have been investigated to control the size and shape of CdS QDs.

CdS QDs were fabricated via numerous fabrication routes in various media; these routes include soft templates (such as liquid crystals, polymers, micelles [1012]), sonochemical [13], capping agent [14], multi phase [15] or coordination complexes [16] in order to control the size and morphology of CdS QDs. Other important fabrication routes based on block copolymers, hyper-branched polymers and dendrimers, were also used for the fabrication of CdS QDs. These special polymers act as nanoreactors as well as stabilizers for CdS QDs in order to restrict their diffusion and growth $[17,18]$. First, cadmium ion is stabilized by the polymer matrix; then after sulfurization, obtained CdS QDs are stabilized without any agglomeration [18]. Stabilization of metal sulphide QDs in a polymer matrix ultimately forms a nanocomposite. The final properties of this nanocomposite correspond to the combination of those of the dispersed QDs and the polymer matrix. This polymer based approach provides an opportunity to improve the optical and electrical properties of nanoscale CdS [19]. Among the above fabrication routes; capping-assisted fabrication is very promising for the development of diversified semiconducting QDs. It is well known that the final morphology of nanocrystals or QDs is largely depended on the type of capping agent absorbed on their surfaces [20, 21]. Among the various capping agents, oleic acid (OA) is one of the well-known capping agents for various QDs. The nature of binding of OA depends on the synthesis route used and in the most of these routes, OA is chemisorbed as carboxylate onto QDs surface leading to good stability and better size control [22].

In this paper, we report a simple, cost effective and straightforward non-aqueous synthesis route to develop OA-capped CdS QDs without using any complicated 


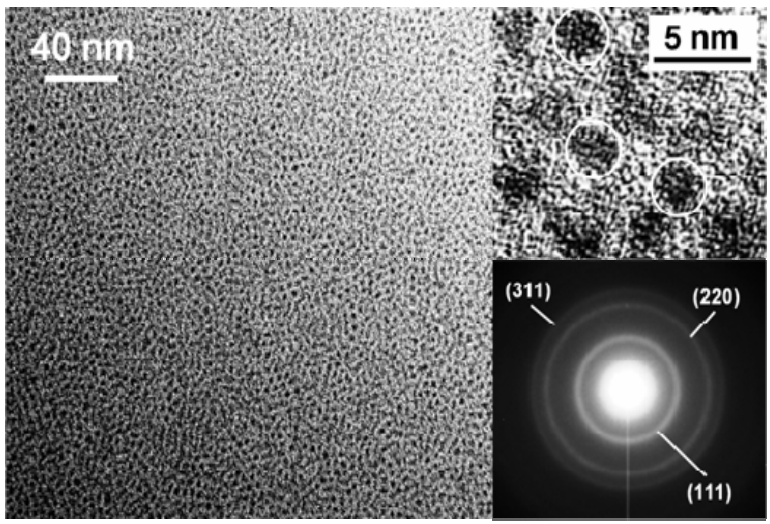

Figure 1. TEM images (at two different magnifications) of OA-capped CdS QDs along with SAED.

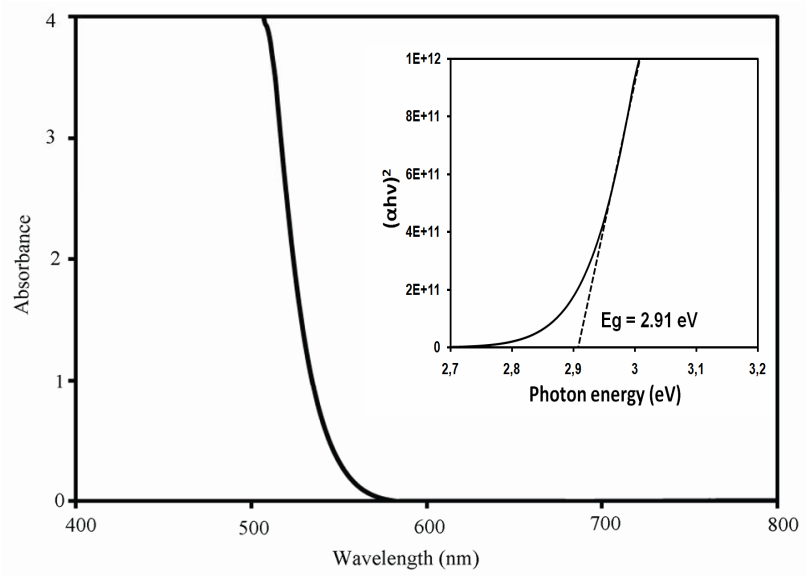

Figure 2. Absorption spectrum of OA-capped CdS QDs. The inset shows the plot of $(\alpha \mathrm{hv})^{2}$, as a function of photon energy, used to determine the energy band gap.

reagents compared to previously reported fabrication routes $[15,23]$. Temperature used throughout this process is lower than that already used for CdS synthesis ( $200^{\circ} \mathrm{C}$ ). Compared to aqueous fabrication techniques, non-aqueous fabrication medium allows the re-dispersion of OA-capped CdS QDs in other non-polar solvents for their further utilization in different applications, including nano-electronics.

\section{Experimental}

CdS QDs were prepared through thermal reaction of OA stabilised $\mathrm{Cd}^{+2}$ and thioacetamide in hot n-hexane media. A $0.05 \mathrm{mmol}(266 \mathrm{mg})$ of cadmium acetate dihydrate was mixed with $1.0 \mathrm{ml}$ of $\mathrm{OA}$ and $20 \mathrm{ml}$ of n-hexane. The mixture was then heated at $140^{\circ} \mathrm{C}$ under continuous stirring in an oil bath using water condenser. After 25 minutes of heating, $0.05 \mathrm{mmol}(75 \mathrm{mg})$ of thioacetamide were added to the heated mixture. After 10 minutes of

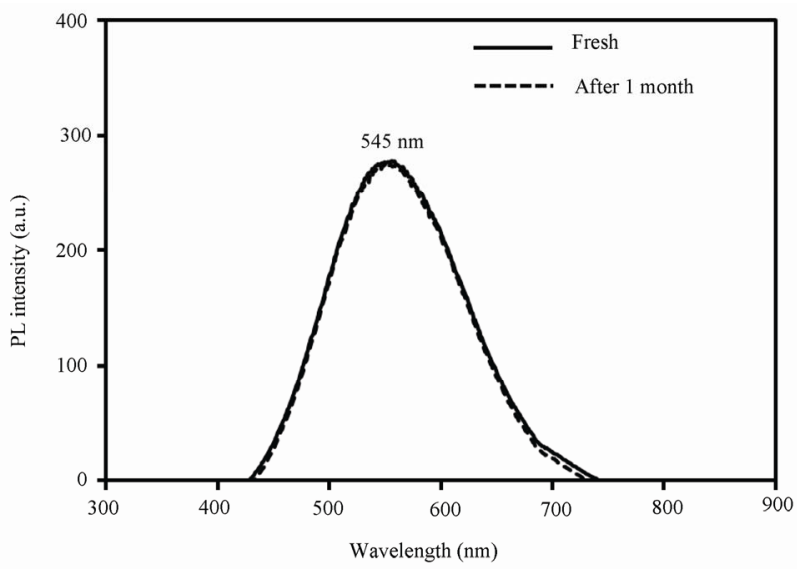

Figure 3. PL spectrum of OA-capped CdS QDs at room temperature.

additional heating, the colorless solution was converted into transparent lemon yellow color, as shown in Figure 4, due to formation of CdS QDs.

\section{Results and Discussion}

Figure 1 shows transmission electron microscopic (TEM) images, along with selective area electron diffraction (SAED) pattern, of synthesized OA-capped CdS QDs. TEM images confirm that CdS QDs are monodispersed and spherical in shape with an average diameter of about $2.6 \mathrm{~nm}$. It is well known that one of the main reasons of QDs agglomeration is their high surface energy at small dimension. However, the absence of agglomeration in our case is due to the effective coating of CdS by OA. The SAED pattern shows a set of concentric rings corresponding to (111), (220) and (311) planes of the cubic CdS phase (JCPDS card No. 75-1546).

The optical absorption of OA-capped CdS QDs (colloidal solution), reported in Figure 2, was studied for a wavelength range of $300-800 \mathrm{~nm}$. The absorption spectrum shows that CdS QDs absorb sharply (from $484 \mathrm{~nm}$ ) in the visible region, as previously reported [23]. This is due to their high monodispersity. The fundamental absorption, which corresponds to electron excitation from valance to conduction bands, was used to determine the nature and the value of the optical band gap. As shown in Figure 2, the estimated band gap of CdS QDs is around $2.91 \mathrm{eV}$, and, compared to that of bulk CdS crystals (2.4 $\mathrm{eV})$, it shows a blue shift because of size quantization effect. Also, the fabrication of this size quantized CdS QDs is very difficult compared to other metal sulphides, such as $\mathrm{PbS}$, because of their small excitonic Bohr radius [24].

Figure 3 shows the photoluminescence (PL) spectrum at room temperature of OA-capped CdS QDs upon exci- 


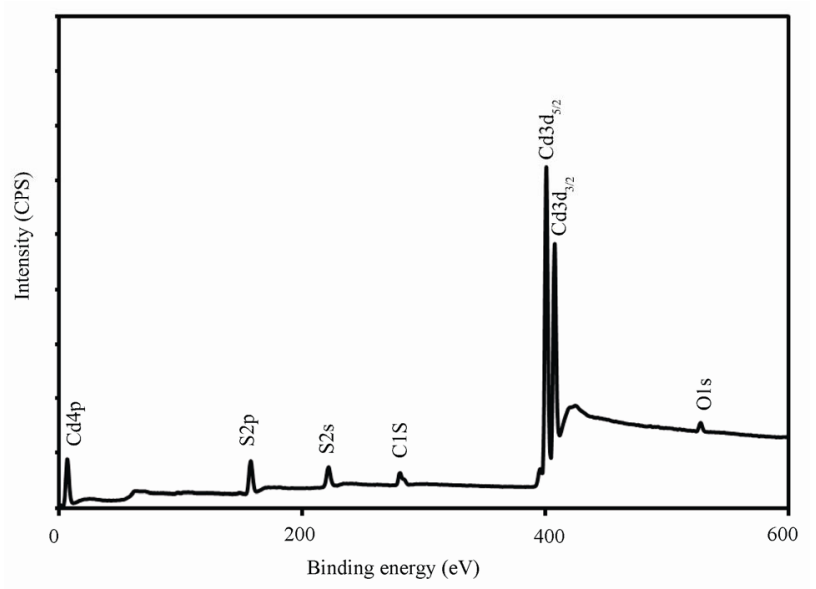

(a)

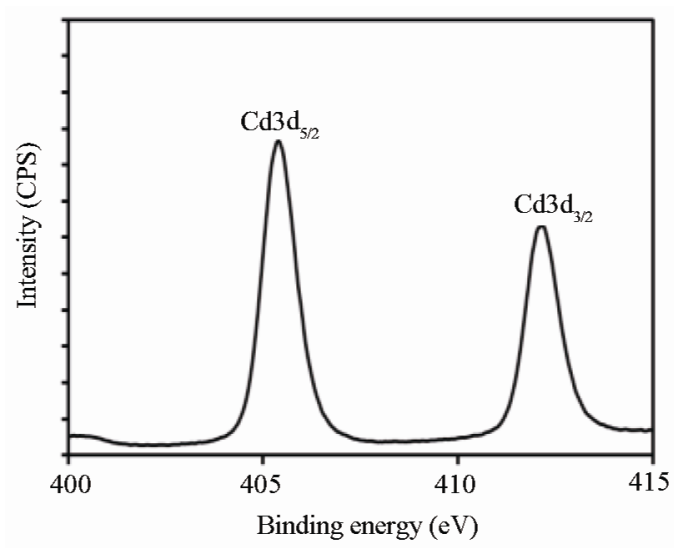

(b)

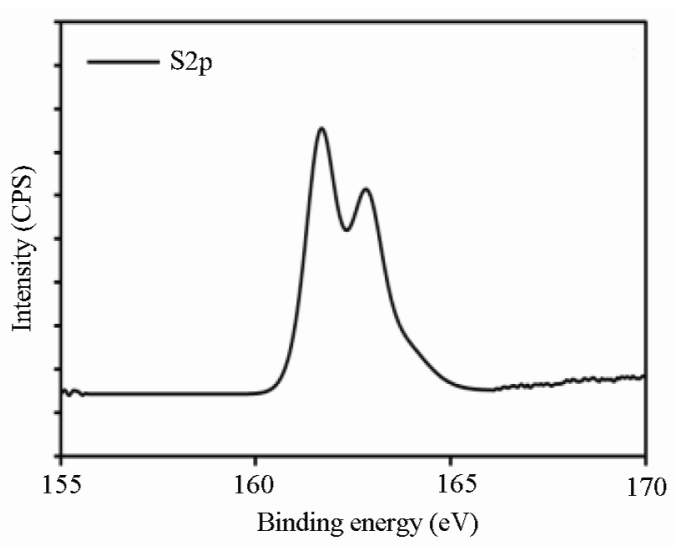

(c)

Figure 4. XPS analysis of OA-capped CdS QDs: (a) a typical XPS survey scan; (b) HR XPS scans for Cd 3d core; (c) HR XPS scans for $S$ 2p core.

tation at $420 \mathrm{~nm}$. As shown, CdS QDs have a broad emission with a maximum value at around $545 \mathrm{~nm}$. It is well known that, for the II-VI capped QDs, the optically excited charge carrier emits light by band-to-band and band-to-defect recombination processes [25]. The latter is mostly driven by the defects that are generated in the gap by surface states and most of the capped CdS QDs fluoresces through this process. In such case, the emission band is normally observed between 400 to $800 \mathrm{~nm}$ and is almost $1.0 \mathrm{eV}$ below the energy band gap of most common QDs [26]. Therefore, in our case, OA-capped CdS QDs emit light between 400 to $750 \mathrm{~nm}$ due to bandto-defects recombination. Due to their core-shell structure, these QDs emit light close to their energy band gap, with a narrow range emission, due to strongly confined photocarriers in the core [25]. After one month, OAcapped CdS QDs shows same luminescence upon excitation at $420 \mathrm{~nm}$ (Figure 3). Stable luminescence indicates that OA-capped CdS QDs are highly stable without any agglomeration.

In order to check their purity and composition, the synthesized OA-capped CdS QDs were characterized by X-ray photoelectron spectroscopy (XPS). The corresponding XPS spectra are shown in Figure 5. As shown in Figure 4(a), the XPS survey scan shows no peaks other than those corresponding to Cd, S, C and O. This confirms the high purity of the synthesized CdS QDs. Highresolution (HR) XPS spectra for $\mathrm{Cd} 3 \mathrm{~d}$ and $\mathrm{S} 2 \mathrm{p}$ regions are shown in Figures 4(b) and 4(c), respectively. All binding energy values were charge-corrected to the $\mathrm{C}$ 1s signal (285.0 eV). As shown in Figure 4(a), the Cd 3d core is split into Cd 3d5/2 and Cd 3d3/2 peaks at 405.3 and $411.9 \mathrm{eV}$, respectively. Figure 4(c) shows two peaks at 161.7 and $162.9 \mathrm{eV}$, corresponding respectively to $\mathrm{S}$ 2 p3/2 and S 2p1/2 of S 2p transitions. All the observed binding energy values are in good agreement with those previously reported for pure CdS [27]. Also, Figure 4(a) confirms the absence of $\mathrm{CdO}$ in the synthesized OAcapped CdS QDs. The Cd 3d5/2 and S 2p3/2 peak areas were used to calculate the elemental ratio of $\mathrm{Cd}$ and $\mathrm{S}$. This ratio was approximately $1: 1$. The additional observed peaks for $\mathrm{C}$ and $\mathrm{O}$ are possibly due to the absorption of these elements on the surfaces of CdS QDs because of their exposure to the air.

Thermogravimetric analysis (TGA) was used in this study in order to check the quality of OA capping of CdS QDs. Figure 5 shows the TGA curve of the synthesized CdS QDs heated from 50 to $650^{\circ} \mathrm{C}$ in presence of air. The figure shows no weight loss for temperatures up to $100^{\circ} \mathrm{C}$, which proves the absence of moisture. Weight decrease observed between around $160^{\circ} \mathrm{C}$ to $360^{\circ} \mathrm{C}$ is due to the elimination of the OA skin on the capped CdS QDs. This sharp decrease is an indication of a monolayer OA coating of CdS QDs surface. At higher temperatures, further decrease in mass is due to the oxidation of CdS 


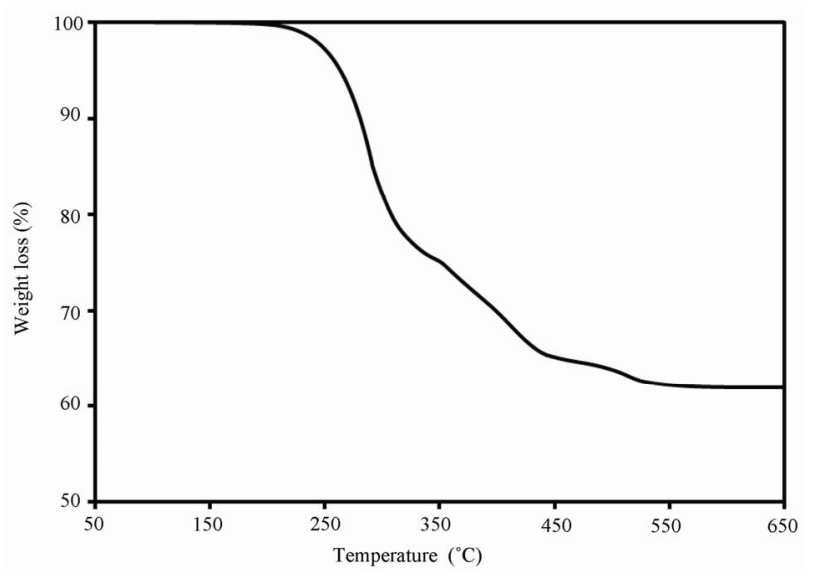

Figure 5. Thermogravimetric pattern of OA-capped CdS QDs.

QDs. After $550^{\circ} \mathrm{C}$, no weight loss is observed due to fully conversion of CdS QDs into CdO. The TGA curve shows that OA capped CdS QDs are composed of about $26 \mathrm{wt} \%$ of OA capping shell and $74 \mathrm{wt} \%$ of CdS.

\section{Conclusions}

In conclusion, a novel low temperature non-aqueous synthesis route is used to develop luminescent OAcapped CdS QDs without using any complicated reagents. According to their physical, optical and thermal properties, CdS QDs are cubic in phase, pure, monodispersed and stable with good optical properties. We believe that this synthesis technique can also be used to synthesize low temperature and cost effective series of other metal sulphide QDs, such as PbS, CuS, NiS, ZnS, etc.

\section{Acknowledgements}

The authors would like to thank the Natural Sciences and Engineering Research Council of Canada (NSERC) for financial support of this work.

\section{REFERENCES}

[1] J.-S. Hu, Y.-G. Guo, H.-P. Liang, L.-J. Wan, C.-L. Bai and Y.-G. Wang, "Interface Assembly Synthesis of Inorganic Composite Hollow Spheres,” Journal of Physical Chemistry B, Vol. 108, No. 28, 2004, pp. 9734-9738. doi:10.1021/jp038050o

[2] D. Wu, X. Ge, Z. Zhang, M. Wang and S. Zhang, "Novel One-Step Route for Synthesizing CdS/Polystyrene Nanocomposite Hollow Spheres,” Langmuir, Vol. 20, No. 13, 2004, pp. 5192-5195. doi:10.1021/la049405d

[3] B. Ludolph, M. A. Malik, P. O’Brien and N. Revaprasadu, "Novel Single Molecule Precursor Routes for the Direct Synthesis of Highlymonodispersed Quantum Dots of Cadmium or Zinc Sulfide or Selenide,” Chemical Communication, No. 17, No. 30, 1998, pp. 1849-1850.

\section{doi:10.1039/A805411H}

[4] H. Hu, S.-C. Kung, L.-M. Yang, M. E. Nicho and R. M Penner, "Photovoltaic Devices Based on ElectrochemicalChemical Deposited CdS and Poly3-octylthiophene Thin Films," Solar Energy Materials and Solar Cells, Vol. 93, No. 1, 2009, pp. 51-54. doi:10.1016/j.solmat.2008.03.011

[5] R. M. Ma, L. Dai, H. B. Huo, W. Q. Yang, G. G. Qin, P. $\mathrm{H}$ Tan, C. H. Huang and J. Zheng, "Synthesis of High Quality N-Type CdS Nanobelts and Their Applications in Nanodevices,” Applied Physics Letters, Vol. 89, No. 20, 2006, p. 203120. doi:10.1063/1.2387982

[6] J. He, W. Ji, G. H. Ma, S. H. Tang, E. S. W. Kong, S. Y. Chow, X. H. Zhang, Z. L. Hua and J. L. Shi, "Ultrafast and Large Third-Order Nonlinear Optical Properties of CdS Nanocrystals in Polymeric Film,” Journal of Physical Chemistry B, Vol. 109, No. 10, 2005, pp. 4373-4376. doi:10.1021/jp047787q

[7] K. Sato, Y. Tachibana, S. Hattori, T. Chiba and S. Kuwabata, "Polyacrylic Acid Coating of Highly Luminescent CdS Nanocrystals for Biological Labeling Applications," Journal of Colloid Interface Science, Vol. 324 No. 1-2, 2008, pp. 257-260. doi:10.1016/j.jcis.2008.04.075

[8] Y. Guo, H. Zhang, Y. Wang, Z. L. Liao, G. D. Li and J. S. Chen, "Controlled Growth and Photocatalytic Properties of CdS Nanocrystals Implanted in Layered Metal Hydroxide Matrixes,” Journal of Physical Chemistry B, Vol. 109, No. 24, 2005, pp. 21602-21607. doi:10.1021/jp054400q

[9] S. Rengaraj, S. H. Jee, S. Venkataraj, Y. Kim, S. Vijayalakshmi, E. Repo, A. Koistinen and M. Sillanpaa, "CdS Microspheres Composed of Nanocrystals and Their Photocatalytic Activity,” Journal of Nanoscience and Nanotechnology, Vol. 11, No. 3, 2011, pp. 2090-2099. doi:10.1166/jnn.2011.3760

[10] C. Tura, N. Coombs and O. Dag, "One-Pot Synthesis of CdS Nanoparticles in the Channels of Mesosructured Silica Films and Monoliths," Chemistry of Materials, Vol. 17, No. 3, 2005, PP. 573-579. doi:10.1021/cm048484b

[11] Y. Zhang, Y. Chen, H. Niu and M. Gao, "Formation of CdS Nanoparticle Necklaces with Functionalized Dendronized Polymers," Small, Vol. 2, No. 11, 2006, pp. 1314-1319. doi:10.1002/smll.200600067

[12] W. Xu and D. L. Akins, "Reverse Micellar Synthesis of CdS Nanoparticles and Self-Assembly into a Superlattice," Materials Letters, Vol. 58, No. 21, 2004, pp. 2623-2626. doi:10.1016/j.matlet.2004.03.035

[13] N. Ghows and M. H. Entezari, "Anovel Method for the Synthesis of CdS Nanoparticles without Surfactant," Ultrasonics Sonochemical, Vol. 18, No. 1, 2011, pp. 269275. doi:10.1016/j.ultsonch.2010.06.008

[14] B. S. Amma, K. Ramakrishna and M. Pattabi, "Comparison of Various Organic Stabilizers as Capping Agents for CdS Nanoparticles Synthesis," Journal of Materials Science: Materials in Electronics, Vol. 18, No. 11, 2007, pp. 1109-1113. doi:10.1007/s10854-007-9139-2

[15] D. Pan, S. Jiang, L. An and B. Jiang, “Controllable Syn- 
Simple Non-Aqueous Fabrication Route for Oleic Acid Capped Luminescent Cadmium Sulphide Quantum Dots at Relatively 65 Low Temperature

thesis of Highly Luminescent and Monodisperse CdS Nanocrystals by a Two-Phase Approach under Mild Conditions," Advance Materials, Vol. 16, No. 2004. pp. 982985. doi:10.1002/adma.200400010

[16] T. Mandal, V. Stavila, I. Rusakova, S. Ghosh and K. H. Whitmire, "Molecular Precursors for CdS Nanoparticles: Synthesis and Characterization of Carboxylate-Thiourea or Thiosemicarbazide Cadmium Complexes and Their Decomposition,” Chemistry Materials, Vol. 21, No. 23, 2009, pp. 5617-5626. doi:10.1021/cm902230u

[17] L. Qi, H. Colfen and M. Antonietti, "Synthesis and Characterization of CdS Nanoparticles Stabilized by Double-Hydrophilic Block Copolymers," Nano Letters, Vol. 1, No. 2, 2001, pp. 61-65. doi:10.1021/nl0055052

[18] B. I. Lemon and R. M. Crooks, "Preparation and Characterization of Dendrimer-Encapsulated CdS Semiconductor Quantum Dots,” Journal of American Chemical Society, Vol. 122, No. 51, 2000, pp. 12886-12887. doi:10.1021/ja0031321

[19] R. B. Cheyne and M. G. Moffitt, "Hierarchical Nanoparticle/Block Copolymer Surface Features via Synergistic Self-Assembly at the Air-Water Interface," Langmuir, Vol. 21, No. 23, 2005, pp. 10297-10300. doi:10.1021/la0519397

[20] F. Chen, R. J. Zhou, L. G. Yang, N. Liu, M. Wang and H. Z. Chen, "Large-Scale and Shape-Controlled Syntheses of Three-Dimensional CdS Nanocrystals with Flowerlike Structure," Journal of Physical Chemistry C, Vol. 112, No. 4, 2008, pp. 1001-1007. doi:10.1021/jp709699h

[21] C.-C. Kang, C.-W. Lai, H.-C. Peng, J.-J. Shyue and P.-T. Chou, "2D Self-Bundled CdS Nanorods with Micrometer Dimension in the Absence of an External Directing Process,” ACS Nano, Vol. 2, No. 4, 2008, pp. 750-756.

\section{doi:10.1021/nn800020h}

[22] M. V. Limaye, S. B. Singh, S. K. Date, D. Kothari, V. Raghavendra Reddy, A. Gupta, V. Sathe, R. J. Choudhary and S. K. Kulkarni, "High Coercivity of Oleic Acid Capped $\mathrm{CoFe}_{2} \mathrm{O}_{4}$ Nanoparticles at Room Temperature," Journal of Physical Chemistry C, Vol. 113, No. 27, 2009, pp. 9070-9076.

[23] M. Tamborra, M. Striccoli, R. Comparelli, M.L. Curri, A. Petrella and A.gostiano, "Optical Properties of Hybrid Composites Based on Highly Luminescent CdS Nanocrystals in Polymer," Nanotechnology, Vol. 15, No. 4, 2004, pp. S240-S244. doi:10.1088/0957-4484/15/4/023

[24] A. Henglein, "Small-Particle Research: Physico-Chemical Properties of Extremely Small Colloidal Metal and Semiconductor Particles,” Chemical Reviews, Vol. 89, No. 8, 1989, pp. 1861-1873. doi:10.1021/cr00098a010

[25] M. N. Kalasand, M. K. Rabinal and B. G. Mulimani, "Facile Synthesis of Bioconjugated Fluorescent CdS NaNoparticles of Tunable Light Emission,' Journal of Physics D: Applied Physics, Vol. 43, 2010, p. 305301. doi:10.1088/0022-3727/43/30/305301

[26] H. Li, W. Y. Shih and W.-H. Shih, "Synthesis and Characterization of Aqueous Carboxyl-Capped CdS Quantum Dots for Bioapplications,” Industrial \& Engineering Chemistry Research, Vol. 46, No. 7, 2007, pp. 2013-2019. doi:10.1021/ie060989j

[27] S. Rengaraj, S. Venkataraj, S. H. Jee, Y. Kim, C.-W. Tai, E. Repo, A. Koistinen, A. Ferancova and M. Sillanpaa, "Cauliflower-Like CdS Microspheres Composed of Nanocrystals and Their Physicochemical Properties," Langmuir, Vol. 27, No. 1, 2011, pp. 352-358. doi:10.1021/la1032288 